\title{
Constraint-based Equilibrium and Stiffness Control of Variable Stiffness Actuators
}

\author{
Matthew Howard, David J. Braun and Sethu Vijayakumar
}

\begin{abstract}
Considerable research effort has gone into the design of variable passive stiffness actuators (VSAs). A number of different mechanical designs have been proposed, aimed at either a biomorphic (i.e., antagonistic) design, compactness, or simplified modelling and control. In this paper, we propose a (model-based) unified control methodology that is able to exploit the benefits of variable stiffness independent of the specifics of the mechanical design. Our approach is based on forming constraints on commands sent to the VSA to ensure that the equilibrium position and stiffness of the VSA are tracked to the desired values. We outline how our approach can be used for tracking stiffness and equilibrium position both in joint and task space, and how it may be used in the context of constrained local optimal feedback control. In our experiments we illustrate the utility of our approach in the context of online teleoperation, to transfer compliant human behaviour to a variable stiffness device.
\end{abstract}

\section{INTRODUCTION}

In recent years, considerable research effort has gone into the design of variable passive stiffness actuators (VSAs). A number of designs have been proposed, directed at different applications, each with their own benefits and disadvantages. For example, several designs have focused on imitating the human musculoskeletal system [11], often resulting in antagonistic actuation systems. These have the benefit that transferring behaviour from human to robot is relatively simple (e.g., by drawing a correspondence between EMG signals and actuator commands), but the disadvantage that they have complex dynamics and can be hard to build into multi-joint devices. Other proposed designs have focused on simplifying the dynamics (and thereby the control) [5] or improving scalability, e.g., with joint-internal VSA designs [15]. These often have several benefits, such as compactness, but the difficulty then lies in finding appropriate controllers, especially when trying to mimic the capabilities of humans [4] and exploit the benefits of variable stiffness.

In general, the final choice of VSA mechanism for a particular robotic device will depend on many different factors related to the specific application. However, if we can find an appropriately general control framework that can be applied to a number of different designs, we can ease this design decision. In other words, we would like to find a unified control methodology, that is able to exploit the benefits of variable stiffness independent of the specifics of the mechanical design.

In previous work, several approaches have been suggested to achieve this goal. For example, De Luca et al. [10] proposed using inverse dynamics and feedback linearisation in order to cancel out non-linearities and track joint stiffness and equilibrium position profiles. They showed that, assuming sufficiently smooth reference trajectories (differentiable

M. Howard, D. J. Braun and S. Vijayakumar are with the Institute of Perception Action and Behaviour, University of Edinburgh, Scotland, UK. E-mail: matthew.howardeed.ac.uk up to 4th order), and assuming a diagonal stiffness matrix, these profiles can be tracked in a similar way to that used for position and torque control. Albu-Schaeffer et al. [1] used a similar approach with the introduction of torque feedback terms to improve disturbance rejection, with demonstrations of the method on a 7-axis robot arm.

More recently, Tahara et al. [13] suggested an approach for resolving redundancy in the actuation of antagonistically actuated systems, with a view to understanding the control of the human musculoskeletal system. Their approach was based on defining Cartesian-space force controllers with virtual spring dynamics, and using the inverse mapping from Cartesian space to joint space, and then on to the muscle space, in order to find appropriate muscle activations to realise the desired movement. In this case, stiffness was implicitly controlled according to the choice of the redundant internal forces.

In this paper, we propose a novel constraint-based framework that allows us to control equilibrium position and stiffness of an arbitrary VSA, given appropriate information about the actuator dynamics. The approach is similar to popular constraint-based schemes in kinematic [9], or torque control [12], but is applicable to redundantly actuated robotic VSA devices to give accurate, closed loop tracking of desired stiffness and position profiles. In addition, our approach allows us to design (i) hierarchical controllers where actuation redundancy can be explicitly resolved in a prioritised framework, (ii) controllers in which constraints on stiffness can be imposed (e.g., enforcing a particular stiffness profile for safety reasons). Furthermore, our approach can also be used for assessing the benefits of VSAs over conventional fixed-stiffness actuators, as we will show in the context of constrained optimal feedback control. In our experiments we test our approach for tracking pre-specified equilibrium position and stiffness profiles on a number of different simulated VSAs, and in an online teleoperation task on hardware using a MACCEPA joint [5].

\section{Problem Definition}

Our aim is to derive joint stiffness and equilibrium position controllers for VSAs, independently of the mechanism used for varying stiffness. For example, we may have a control law determining a stiffness profile for one VSA, (e.g., the MACCEPA, Fig. 1(b)) and wish to transfer it to a second, different VSA (e.g., the Edinburgh SEA, Fig. 1(c)) for comparison. Alternatively, we may wish to transfer the stiffness from a human, measured during some task, to reproduce the same compliant behaviour on a robotic device.

Specifically, we assume that the VSA that we wish to control has state $\mathbf{x} \in \mathbb{R}^{p}$ (e.g., joint positions $\mathbf{q} \in \mathbb{R}^{n}$ and velocities $\dot{\mathbf{q}} \in \mathbb{R}^{n}$ ), and applying command $\mathbf{u} \in \mathbb{R}^{m}$ results in a joint torque of the form 


$$
\tau=\tau(\mathbf{x}, \mathbf{u})
$$

which, for a variable stiffness actuator, may be re-written in the form

$$
\boldsymbol{\tau}(\mathbf{x}, \mathbf{u})=-\mathbf{K}(\mathbf{x}, \mathbf{u})\left(\mathbf{q}-\mathbf{q}_{0}(\mathbf{x}, \mathbf{u})\right)
$$

where $\mathbf{K}(\mathbf{x}, \mathbf{u}) \in \mathbb{R}^{n \times n}$ is the joint stiffness matrix and $\mathbf{q}_{0}(\mathbf{x}, \mathbf{u}) \in \mathbb{R}^{n}$ are the equilibrium positions of the joints. Note that both of these quantities may, in general, have nonlinear dependence on $\mathbf{x}$ and $\mathbf{u}$, depending on the design of the mechanism.

Our goal is to derive appropriate commands $\overline{\mathbf{u}}$ that realise a desired stiffness $\mathbf{K}_{d}$ and equilibrium position $\mathbf{q}_{0, d}$. These may be given either as fixed values (e.g., enforcing a fixed stiffness for the joint) or as variables to be tracked (e.g., in online tracking of equilibrium position and stiffness in a teleoperation task). Alternatively, we may wish to prioritise control of the position over that of stiffness. For example, in a punching task, we may want a high stiffness at the time of impact (for a hard punch), but since the first priority is to hit the target, we may have to sacrifice some stiffness in order to achieve that goal. The extent to which this sacrifice needs to be made will depend on the design of the variable stiffness mechanism, and any coupling that exists between position and stiffness. In the next section, we clarify these issues with reference to different example implementations of variable stiffness actuation.

\section{Example: Ideal VSA, MACCEPA and Edinburgh SEA}

To illustrate the influence that different mechanical designs have on the control of stiffness and equilibrium position, we consider three possible designs for a single-joint VSA.

The first and simplest of the three, is the idealised VSA (see Fig. 1(a)), in which we assume that the stiffness and equilibrium position are directly controllable in the command vector, i.e., $\mathbf{u}=\left(q_{0}, k\right)^{T}$. In this case, the control of equilibrium position and stiffness is exactly orthogonal, enabling use to select any combination of position and stiffness. This is illustrated in Fig. 1(a), right panel, where, for example, moving along the $y$-axis (corresponding to $u_{2}$ ) adjusts the stiffness, but has no effect on the equilibrium position, and vice versa. Unfortunately, in real mechanisms it is rarely possible to achieve such ideal behaviour.

In contrast, consider the MACCEPA [5] and the Edinburgh SEA [11] as examples of actuators of competing designs, that have both been realised in hardware. For the MACCEPA, the applied joint torque (2) is given by

$$
\tau(\mathbf{x}, \mathbf{u})=\kappa B C \sin \alpha\left(1+\frac{r u_{2}-(C-B)}{\sqrt{B^{2}+C^{2}-2 B C \cos \alpha}}\right)
$$

where $\mathbf{x}=(q, \dot{q})^{T}, \alpha=u_{1}+q, \kappa$ is the spring constant, $B$ and $C$ are the distances illustrated in Fig. 1(b) and $r$ is the radius of the winding drum (mounted on the servo that extends the spring). Note that, due to the multiplication of terms dependent on $u_{1}$ and $u_{2}$, there exists a coupling between equilibrium position and stiffness. In particular, while the equilibrium position is only influenced by controlling the angle of the lever arm $\left(u_{1}\right)$, away from equilibrium the stiffness is influenced both by the pre-tensioning of the spring $\left(u_{2}\right)$ as well as the lever arm angle $\left(u_{1}\right)$. To illustrate this, we may make a similar plot of the equilibrium position and stiffness as a function of motor commands as for the ideal VSA (ref Fig. 1, middle row). In this case we can see that, though the equilibrium position is only influenced by the position of the first motor $\left(u_{1}\right)$, there is a rather complex, non-linear relationship between the motor commands and joint stiffness, making independent control of stiffness difficult.

A similar argument applies to control of the Edinburgh SEA [11], for which the torque relationship is

$$
\tau(\mathbf{x}, \mathbf{u})=-\hat{\mathbf{z}}^{T}\left(\mathbf{a} \times \mathbf{F}_{1}-\mathbf{a} \times \mathbf{F}_{2}\right)
$$

where $\hat{\mathbf{z}}$ is the unit vector along the joint rotation axis, $\mathbf{a}=(a \cos q, a \sin q, 0)^{T}, \mathbf{F}_{i}=\kappa\left(s_{i}-s_{0}\right) \frac{\mathbf{s}_{i}}{s_{i}}, i \in\{1,2\}$ are the forces due to the two springs (both with spring constant $\kappa), \mathbf{s}_{1}=\left(-h-L \sin u_{1},-d+L \cos u_{1}, 0\right)^{T}+\mathbf{a}$ and $\mathbf{s}_{2}=\left(h+L \sin u_{2},-d+L \cos u_{2}, 0\right)^{T}-\mathbf{a}$ are the extensions of the two springs, and all other quantities are illustrated in Fig. 1(c). In this case, due to the antagonistic actuation, there is a strongly coupled, non-linear relationship between the motor commands and the joint equilibrium position and stiffness (as illustrated in Fig. 1(c), right) making it difficult to control these quantities directly.

As illustrated by these examples, it is clear that even for relatively simple VSA designs, there is considerable difficulty in directly regulating the position and stiffness. At first glance, it would seem that, in order to exploit the dynamic properties of these actuators, it would be necessary to develop specialised controllers for each design. However, in the next section we will outline a general method for controlling arbitrary VSAs with a constraint-based framework.

\section{METHOD}

Motivated by the examples in the preceding section, here we outline our method for constraint-based control of equilibrium position and stiffness. We will first outline the basic approach, and then illustrate how such an approach can be used in the context of constrained local optimal feedback control.

\section{A. Model-based Equilibrium and Stiffness Prediction}

Our approach is a model based control scheme in which we assume that we have knowledge of the relationship between the robot state $\mathbf{x} \in \mathbb{R}^{p}$ (e.g., $\mathbf{x}=(\mathbf{q}, \dot{\mathbf{q}})^{T} \in \mathbb{R}^{2 n}$ ), the command vector $\mathbf{u} \in \mathbb{R}^{m}$, and the resultant joint torque $\boldsymbol{\tau}(\mathbf{x}, \mathbf{u})$, either in closed form or as a non-parametric model (e.g., from non-parametric regression). Note that, in general, variable stiffness actuators are redundantly actuated (since they have at least one additional degree of freedom per joint for changing the stiffness), so $m>n$.

Given (1) we can derive an expression for the equilibrium position vector as a function of state and command

$$
\mathbf{q}_{0}=\mathbf{q}_{0}(\mathbf{x}, \mathbf{u}) \in \mathbb{R}^{n}
$$

by solving $\boldsymbol{\tau}(\mathbf{x}, \mathbf{u})=\mathbf{0}$ for $\mathbf{q}$. This may be derived analytically, or calculated numerically with a root-finding algorithm (e.g., the Newton-Raphson method).

Also using (1), we can derive the joint stiffness matrix

$$
\mathbf{K}=\mathbf{K}(\mathbf{x}, \mathbf{u})=-\left.\frac{\partial \boldsymbol{\tau}(\mathbf{x}, \mathbf{u})}{\partial \mathbf{q}}\right|_{\mathbf{q}} \in \mathbb{R}^{n \times n}
$$

Again, in many cases we may obtain (6) in closed analytical form, or alternatively use numerical finite differences. For 

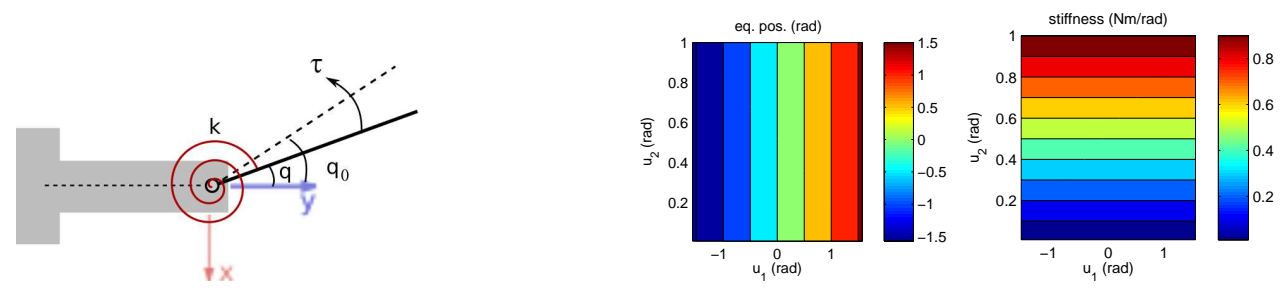

(a) Ideal 1-link VSA.
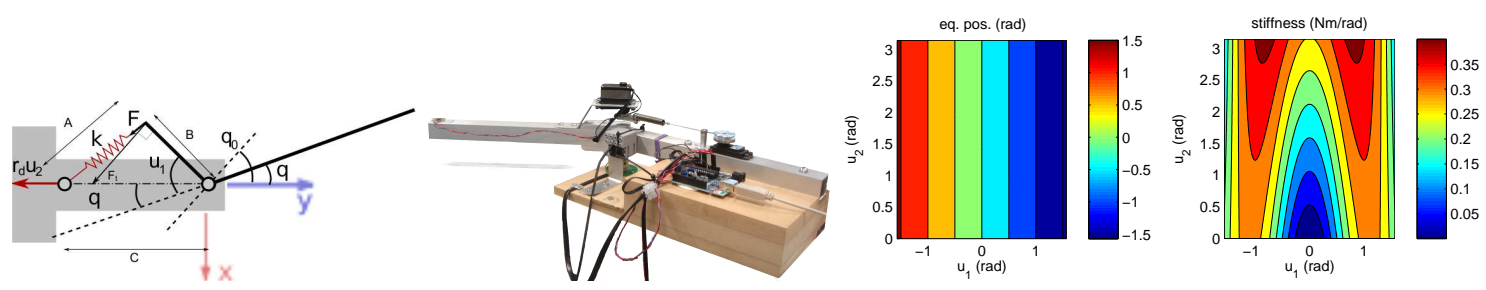

(b) MACCEPA.
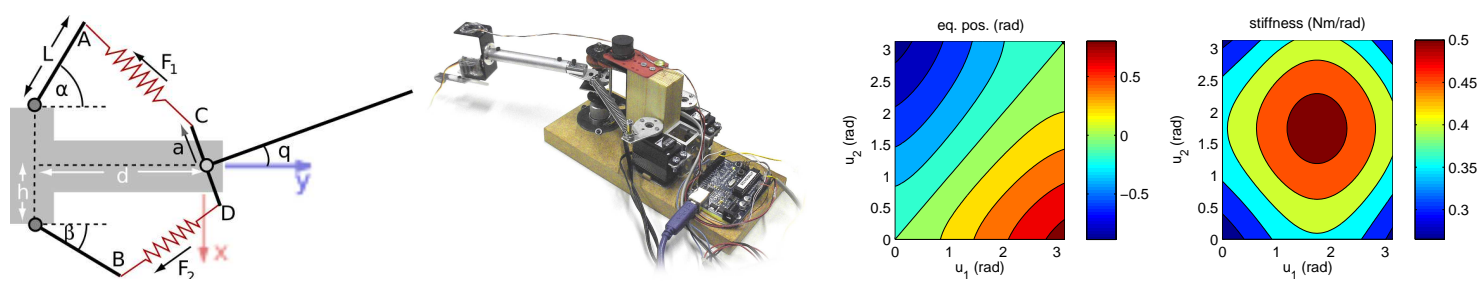

(c) Edinburgh SEA.

Fig. 1. Left: Geometry, dynamics and hardware implementation of the 1-link variable stiffness actuators used in the experiments. Right: Equilibrium position and stiffness as a function of commands $\mathbf{u}$ (evaluated at $q=0, \dot{q}=0$ ).

convenience, we can write the stiffness in vector form as $\mathbf{k}(\mathbf{x}, \mathbf{u})=\operatorname{vec}(\mathbf{K}(\mathbf{x}, \mathbf{u})) \in \mathbb{R}^{n^{2}}$.

Note that in general (5) and (6) are non-linear functions of the state and commands. Note also that, depending on the system, the dimensionality of $\mathbf{k}(\mathbf{x}, \mathbf{u})$ may vary. For example, the stiffness of each joint may be coupled so that $\mathbf{K}$ is symmetric, or, alternatively, the stiffness of individual joints may be independent (e.g., as would be the case in a chain of MACCEPA actuators). In the latter case, $\mathbf{K}$ reduces to a diagonal matrix and we can omit the off-diagonal elements, resulting in $\mathbf{k} \in \mathbb{R}^{n}$.

\section{B. Resolved Equilibrium and Stiffness Tracking Control}

Having derived (5) and (6) for estimating the equilibrium position and stiffness, we are now in a position to design constraint-based controllers. We note that, in general, for VSAs with an actuation relationship of the form (2), we cannot find a linear, orthogonal decomposition in the direct control space since the multiplication of stiffness with equilibrium position introduces a quadratic dependence on $\mathbf{u}$. For this reason, we must instead move to the command velocity space for control.

In particular, we can take the time derivative of (5) and (6) to find the linearised forward impedance dynamics

$$
\begin{aligned}
\dot{\mathbf{q}}_{0} & =\mathbf{J}_{\mathbf{q}_{0}}(\mathbf{x}, \mathbf{u}) \dot{\mathbf{u}}+\mathbf{P}_{\mathbf{q}_{0}}(\mathbf{x}, \mathbf{u}) \dot{\mathbf{x}} \\
\dot{\mathbf{k}} & =\mathbf{J}_{\mathbf{k}}(\mathbf{x}, \mathbf{u}) \dot{\mathbf{u}}+\mathbf{P}_{\mathbf{k}}(\mathbf{x}, \mathbf{u}) \dot{\mathbf{x}}
\end{aligned}
$$

where $\dot{\mathbf{q}}_{0}, \dot{\mathbf{k}}$ are the change in equilibrium position and stiffness with respect to time, $\dot{\mathbf{u}} \in \mathbb{R}^{n}$ is the rate of change of motor commands, $\mathbf{J}_{\mathbf{q}_{0}} \in \mathbb{R}^{n \times m}$ and $\mathbf{J}_{\mathbf{k}} \in \mathbb{R}^{n^{2} \times m}$ are the Jacobian of the equilibrium position and the stiffness with respect to motor commands, while $\mathbf{P}_{\mathbf{q}_{0}} \in \mathbb{R}^{n \times p}$ and $\mathbf{P}_{\mathbf{k}} \in \mathbb{R}^{n^{2} \times p}$ are the corresponding Jacobian with respect to the state.

To simultaneously control equilibrium position and stiffness, we can invert this relationship to yield ${ }^{1}$

$$
\dot{\mathbf{u}}=\mathbf{J}^{\dagger} \dot{\mathbf{r}}+\left(\mathbf{I}-\mathbf{J}^{\dagger} \mathbf{J}\right) \mathbf{u}_{0}
$$

where $\dot{\mathbf{r}}=\left(\dot{\mathbf{q}}_{0}-\mathbf{P}_{\mathbf{q}_{0}} \dot{\mathbf{x}}, \dot{\mathbf{k}}-\mathbf{P}_{\mathbf{k}} \dot{\mathbf{x}}\right)^{T} \in \mathbb{R}^{n+n^{2}}, \mathbf{J}=$ $\left(\mathbf{J}_{\mathbf{q}_{0}}, \mathbf{J}_{\mathbf{k}}\right)^{T}$ is the combined Jacobian, $\mathbf{I}$ is the identity matrix, $\mathbf{J}^{\dagger}$ denotes the Moore-Penrose pseudoinverse of $\mathbf{J}$ and $\mathbf{u}_{0}$ is an arbitrary vector. The latter can be used to resolve any further redundancy in the actuation (such as additional actuators used for varying damping [8]).

Application of (9) requires state derivatives, provided by feedback, or calculated from the analytical model of the system dynamics. To avoid the requirement on analytical modelling, and also to circumvent the noise and phaselag issues related with the feedback on $\dot{x}$, we employ online feedback about the current stiffness and equilibrium states, i.e., we choose $\dot{\mathbf{r}}$ according to the difference in the desired and actual equilibrium and stiffness values $\dot{\mathbf{r}}=$ $\left(\dot{\mathbf{q}}_{0}^{*}-\dot{\mathbf{q}}_{0}, \dot{\mathbf{k}}^{*}-\dot{\mathbf{k}}\right)^{T}$. This solution is similar to Closed-Loop Inverse Kinematic (CLIK) control [3], and also mitigates instabilities due to constraint drift [2]. For this, since we cannot directly measure the stiffness and equilibrium position on-line, we use (5) and (6) to estimate the current values based on the current estimate of the state.

\footnotetext{
${ }^{1}$ We omit the dependence on $\mathbf{x}$ and $\mathbf{u}$ for readability.
} 


\section{Equilibrium and Stiffness Tracking in Task Space}

The approach described so far can also be extended to equilibrium and stiffness tracking in task (e.g., end-effector) space coordinates. In task space, the restoring force in response to a perturbation is

$$
\mathbf{F}_{s}=-\mathbf{K}_{s}(\mathbf{x}, \mathbf{u}) \delta \mathbf{s} \quad \in \mathbb{R}^{q}
$$

where $\mathbf{s} \in \mathbb{R}^{q}$ is a vector of task space coordinates and $\mathbf{K}_{s} \in \mathbb{R}^{q \times q}$ is the task space stiffness. This force is related to the joint torques through the relationship

$$
\boldsymbol{\tau}=\mathbf{W}(\mathbf{q})^{T} \mathbf{F}_{s}
$$

where $\mathbf{W}(\mathbf{q}) \in \mathbb{R}^{q \times n}$ is the Jacobian from joint to task space $^{2}: \delta \mathbf{s}=\mathbf{W} \delta \mathbf{q}$. Substituting this and (10) into (11), we find

$$
\boldsymbol{\tau}=-\mathbf{W}^{T} \mathbf{K}_{s} \mathbf{W} \delta \mathbf{q}=-\mathbf{K} \delta \mathbf{q} .
$$

Assuming that $\mathbf{W}$ is square and full-rank (i.e., $q=n$ ), by elimination we can then identify the task space stiffness

$$
\mathbf{K}_{s}=\left(\mathbf{W}^{T}\right)^{-1} \mathbf{K} \mathbf{W}^{-1} \text {. }
$$

Similar to the case of joint space stiffness tracking, we can conveniently write $\mathbf{k}_{s}=\operatorname{vec}\left(\mathbf{K}_{s}\right) \in \mathbb{R}^{q^{2}}$ and then derive the task space stiffness Jacobian $\mathbf{J}_{\mathbf{k}_{s}} \in \mathbb{R}^{q^{2} \times m}$ with respect to the motor commands $\mathbf{u}$.

The task space equilibrium position $\mathbf{s}_{0}$ can be found by solving

$$
\mathbf{F}_{s}=\left(\mathbf{W}^{T}\right)^{-1} \boldsymbol{\tau}=\mathbf{0} .
$$

For non-redundant robots $^{3}$, if we have the expression (5), we can calculate $\mathbf{s}_{0}$ directly by mapping the joint space equilibrium position $\mathbf{q}_{0}$ through the forward kinematics function, since at this point $\boldsymbol{\tau}=\mathbf{0}$, which implies $\mathbf{F}_{s}=\mathbf{0}$ through (13). Again the Jacobian $\mathbf{J}_{\mathbf{s}_{0}} \in \mathbb{R}^{q \times m}$ may be derived either in closed form or numerically through finite differences.

For equilibrium position and stiffness tracking in task space, we then take a similar approach to that described in Sec. III-B, replacing $\mathbf{J}_{\mathbf{q}_{0}}, \mathbf{J}_{\mathbf{k}}$ with $\mathbf{J}_{\mathbf{s}_{0}}, \mathbf{J}_{\mathbf{k}_{s}}$ in (9).

\section{Optimal Control with Constrained Stiffness}

Finally, we briefly describe how the framework developed so far can be used in combination with optimal control techniques to place constraints on the change in stiffness.

In general, the optimal control problem is to find a commands $\mathbf{u}^{*}$ that minimise a cost function of the form

$$
J=h(\mathbf{x}(T))+\int_{0}^{T} l(\mathbf{x}, \mathbf{u}, t) d t \quad \in \mathbb{R}
$$

under dynamics of the form

$$
\dot{\mathbf{x}}=\mathbf{f}(\mathbf{x}, \mathbf{u}) \quad \in \mathbb{R}^{p} .
$$

In many cases, we may wish to constrain the optimisation, subject to higher priority considerations, for example, by seeking the optimal controls subject to maintaining a particular stiffness profile for safety reasons. We can impose

\footnotetext{
${ }^{2} \mathrm{We}$ omit the dependence on $\mathbf{q}$ for readability.

${ }^{3}$ Note that, for redundant robots, a similar analysis applies but with the inverses in (12)-(13) replaced by pseudo-inverses. This has the implication that there may exist multiple joint-space equilibria qo for a given task space equilibrium $\mathbf{s}_{0}$.
}

such a constraint using our framework by reformulating the above problem in the command velocity domain.

Specifically, we use the augmented state $\mathbf{y}=\left(\mathbf{y}_{1}, \mathbf{y}_{2}\right)^{T}=$ $(\mathbf{x}, \mathbf{u})^{T} \in \mathbb{R}^{p+m}$, and command $\mathbf{v}=\dot{\mathbf{u}} \in \mathbb{R}^{m}$ and seek the optimal controls $\mathbf{v}^{*}$ in command velocity space with respect to (14) under the constrained dynamics

$$
\dot{\mathbf{y}}=\mathbf{g}(\mathbf{y}, \mathbf{v})=\left(\begin{array}{c}
\mathbf{f}\left(\mathbf{y}_{1}, \mathbf{y}_{2}\right) \\
\mathbf{J}_{k}^{\dagger} \dot{\mathbf{r}}\left(\mathbf{y}_{1}, \mathbf{y}_{2}\right)+\left(\mathbf{I}-\mathbf{J}_{k}^{\dagger} \mathbf{J}_{k}\right) \mathbf{v}
\end{array}\right) \in \mathbb{R}^{p+m}
$$

where $\mathbf{J}_{k}$ is the stiffness Jacobian, $\dot{\mathbf{r}}=\mathbf{k}^{*}-\mathbf{k}\left(\mathbf{y}_{1}, \mathbf{y}_{2}\right)$ and $\mathbf{k}^{*}$ is the desired stiffness. Reformulating the problem in this way ensures that the control sequence is optimised in the null-space of the stiffness Jacobian. This means that whatever control sequence that comes out of the optimisation will have no effect on the stiffness profile. In the experiments we briefly illustrate how such an optimisation can be used in the context of establishing the benefits of variable stiffness designs over a fixed stiffness actuator.

\section{EXPERIMENTS}

In this section, we report numerical simulations and experiments applying our method to the control of several variable stiffness devices. We first illustrate the basic tracking capability of our method on three simulated, singe-joint VSAs given desired equilibrium position and stiffness profiles. We then test the scalability to plants of higher-dimensionality in the context of task-space position and stiffness tracking. We illustrate our method's use in online, interactive control of stiffness in a teleoperation task in hardware. Finally, we illustrate how our method can be applied in the optimal control setting, for analysing the benefits of variable stiffness actuation over traditional fixed-stiffness devices.

\section{A. Basic Tracking Behaviour}

We first test the tracking quality achieved when applying our approach to a number of different VSAs with differing designs in simulation. For this, we apply the approach outlined in Sec. III-B to control the three single-joint VSAs described in Sec. II, namely, the Ideal VSA, the MACCEPA and the Edinburgh SEA to track simple, pre-specified joint equilibrium position and stiffness trajectories. Specifically, the task here is to track a desired stiffness profile of the form

$$
k^{*}(t)=\frac{1}{2} A_{k}\left(\sin \left(\omega_{k} t\right)+1\right)+b_{k}
$$

and equilibrium position

$$
q_{0}^{*}(t)=\frac{1}{2} A_{q_{0}}\left(\sin \left(\omega_{q_{0}} t\right)+1\right)+b_{q_{0}}
$$

where the amplitude $A_{k}=k_{\max }-k_{\min }$ corresponds to the full range of stiffness values (between minimum stiffness $k_{\text {min }}$ and maximum stiffness $k_{\max }$ for the specific actuator) and similarly $A_{q_{0}}=q_{0, \max }-q_{0, \min }$ is the amplitude for the equilibrium position. The offsets $b_{k}=k_{\text {min }}$ and $b_{q_{0}}=q_{0, \text { min }}$ ensure that the desired trajectories stay within the admissible limits of the VSAs. We arbitrarily selected $\omega_{k}=2$ and $\omega_{q_{0}}=$ $3 \mathrm{rad} / \mathrm{s}$. The trajectories were tracked for $4 \mathrm{~s}$, with control at a rate of $50 \mathrm{~Hz}$.

In Fig. 2, we show the tracking performance in terms of the equilibrium position and stiffness, the command sequence generated with the tracking controller (9), and the resultant 

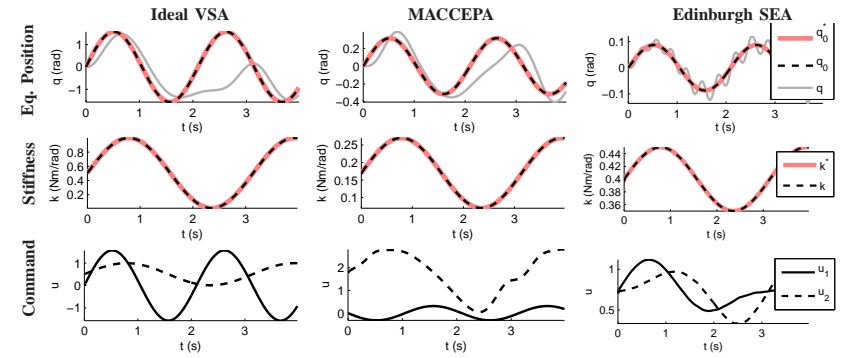

Fig. 2. Simultaneous tracking of sinusoidal equilibrium position and stiffness profiles on the three single-joint VSAs. Top row: desired equilibrium position $q_{0}^{*}$ (light red), realised equilibrium position $q_{0}$ (dashed black) and actual joint position $q$ (medium grey). Middle row: desired stiffness $k^{*}$ (light red) and realised stiffness $k$ (dashed black). Bottom row: Motor commands $u_{1}$ (solid black) and $u_{2}$ (dashed black).

\begin{tabular}{|c|c|c|}
\hline & $R M S E\left(q_{0}, q_{0}^{*}\right)$ & $R M S E\left(k, k^{*}\right)$ \\
\hline Ideal 1-link VSA & 0.000000 & 0.000000 \\
\hline MACCEPA & 0.000000 & 0.000218 \\
\hline Edinburgh SEA & 0.000132 & 0.000308 \\
\hline
\end{tabular}

TABLE I

ERROR IN TRACKED EQUILIBRIUM POSITION AND STIFFNESS FOR THE THREE SIMULATED SINGLE-JOINT VSAS.

trajectory of the joint, for the three VSAs. Looking at the command sequence (top row), we note that the controller generates a different sequence of commands for each of the three VSAs. However, when we look at the equilibrium position (middle row) and stiffness profiles (bottom row) we see that there is good agreement between the realised trajectories $\left(q_{0}, k\right)$ and the desired $\left(q_{0}^{*}, k^{*}\right)$ in all cases. This is confirmed further by the figures for the RMSE between the desired and actual trajectories estimated over the duration of the movement (ref. Table I), which are uniformly low.

It is also interesting to note how, due to the different dynamics of the three plants, the actual position of the joint $q$ lags or oscillates around the commanded equilibrium position, especially when the joint stiffness is very low (around $2 s$ into the movement). This is to be expected since in the low gain control realised here, the response of the system comes as a combined effect of the plant dynamics and the control actions.

\section{B. End-effector Stiffness Tracking on Multi-link Systems}

Our second numerical investigation tests the scalability of our approach for end-effector stiffness tracking (cf. Sec. IIIC) on two higher-dimensional VSA systems. For this, we used (i) an ideal 2-link VSA, and (ii) a biologically plausible model of the human arm with muscle-like actuators (schematic diagrams are provided in Fig. 3). The former can be considered a generalisation of the ideal single-joint VSA, in which the joint torques due to the controls are given by

$$
\tau=-\mathbf{K}\left(\mathbf{q}-\mathbf{q}_{0}\right)
$$

and the equilibrium position and stiffness are directly controllable, i.e., $\mathbf{u}=\left(\mathbf{q}_{0}, \operatorname{vec}(\mathbf{K})\right)^{T}$. The latter is controlled through a system of 6 muscles with Kelvin-Voigt muscle dynamics [7]. Specifically, the control vector $\mathbf{u} \in \mathbb{R}^{6}$ represents muscle activations with a non-linear relationship to the applied torques

$$
\boldsymbol{\tau}=-\mathbf{A}^{T} \mathbf{T}(\mathbf{q}, \dot{\mathbf{q}}, \mathbf{u})
$$

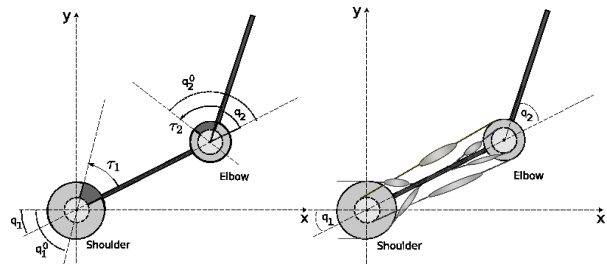

(a) Ideal 2-link VSA. (b) 6-muscle arm model.

Fig. 3. Dynamics models for the two 2-link variable stiffness actuators.

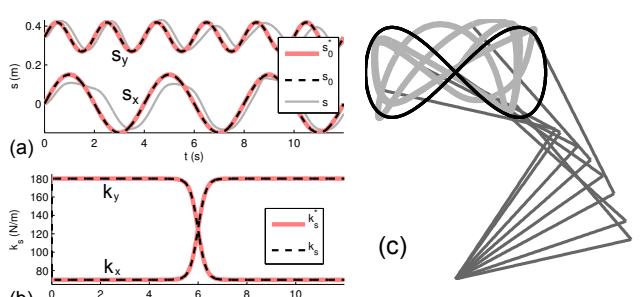

(b) $\mathrm{t}(\mathrm{s})$

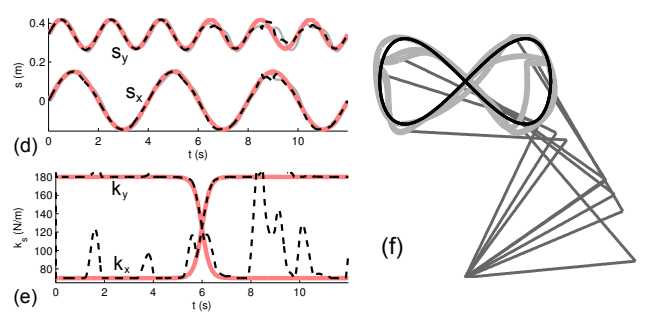

Fig. 4. Tracking of equilibrium position and stiffness profiles in endeffector space on the Ideal 2-DOF VSA (top), and 6-muscle arm model (bottom). Shown are: (a) \& (d) Desired end-effector equilibrium positions $\mathbf{s}_{0}^{*}$ (light red), realised end-effector equilibrium positions $\mathbf{s}_{0}$ (dashed black) and actual end-effector positions s (grey) during the movement, (b) \& (e) Desired end-effector stiffness $\mathbf{k}_{\mathbf{s}}{ }^{*}$ and realised end-effector stiffness $\mathbf{k}_{\mathbf{s}}$, (c) \& (f) stroboscopic plots of the resultant behaviour with the end-effector path shown in light grey.

where $\mathbf{A} \in \mathbb{R}^{2 \times 6}$ is a matrix of moment arms and $\mathbf{T} \in \mathbb{R}^{6}$ are the muscle tensions (for space reasons, we refer the reader to [7] for full details of the model).

The desired equilibrium position trajectory $\mathbf{s}_{0}^{*}(t) \in \mathbb{R}^{2}$ is a figure- 8 in end-effector space, while the desired endeffector stiffness $\mathbf{K}_{s}^{*}(t)$ was designed to switch from lowto high-stiffness in the $x$-direction, and from high to low in the $y$-direction (ref. Fig. 4). Note that, only the diagonal elements of $\mathbf{K}_{s}$ were controlled (i.e., $\mathbf{k}_{s}=\operatorname{diag}\left(\mathbf{K}_{s}\right) \in \mathbb{R}^{2}$ ), the remaining redundancy ${ }^{4}$ was resolved through a null-space policy $\mathbf{u}_{0}=-\alpha\left(\mathbf{u}-\mathbf{u}_{d}\right)$ where $\mathbf{u}$ is the active command, $\mathbf{u}_{d}$ is a default command vector (selected for each of the plants individually) and $\alpha$ is scaling factor. The trajectories were tracked for $12 s$ at a control rate of $150 \mathrm{~Hz}$.

The results are shown in Fig. 4. As can be seen, for the ideal 2-link VSA there is excellent agreement between the desired and tracked equilibrium positions and stiffness (compare light red and dashed black trajectories in Fig. 4(a)(b)). The tracking for the muscle model is also fairly accurate (Fig. 4(d)-(e)), although there are some 'perturbations' away from the desired profiles. Upon examination, we found this to be due to the controller hitting command limits (in the muscle model the commands are constrained such that $\mathbf{u} \geq$

\footnotetext{
${ }^{4}$ Note that, here the full control dimensionality of the two actuators under consideration is $\mathbf{u} \in \mathbb{R}^{6}$, however we only control $\mathbf{s}_{0} \in \mathbb{R}^{2}$ and the diagonal elements $\mathbf{k}_{s} \in \mathbb{R}^{2}$ the effective control is $\mathbf{r}^{*}=\left(\mathbf{s}_{0}, \mathbf{k}_{s}\right)^{T} \in \mathbb{R}^{4}$. This effectively leaves two dimensions of redundancy.
} 
0 , see [7]). This may be alleviated by taking command constraints into account explicitly in the controller design (e.g., using unilateral constraints). However, we note that even without this, the controlled trajectory rapidly converges back to the desired profile when the configuration moves away from these limits.

Looking at the behaviour, (stroboscopic plots in Fig. 4(c), (f)) we see that actual trajectory of the arm behaves like a variable-gain controller. For example, when the stiffness in $x$ is low (first $6 s$ of movement), tracking of the eight suffers in this dimension (ref. light grey line $s_{x}$ in Fig. 4(a)), but is then re-gained the stiffness in $x$ returns to be high (final $6 s$ ). The opposite trend can be observed in the $y$ dimension where stiffness starts high and switches to low. This confirms our expectations about the behaviour under variable end-effector stiffness.

\section{Tracking Human Impedance Profiles}

In this section we illustrate how the proposed approach can be applied to online control of VSAs in an experiment in hardware. For this, we chose to investigate a teleoperation task in which a human operator controls equilibrium position and stiffness of a MACCEPA joint via recordings of his muscle activations. The experimental setup is as follows.

The human operator was fitted with a pair of surface EMG sensors to the wrist extensor and flexor muscles of the forearm (see Fig. 5) that provide streaming data on the activation of the muscles. The raw data was filtered through a band pass filter to remove the lowest and highest frequency components and smooth out noise. The resultant activation data $\mathbf{a}=\left(a_{\text {ext }}, a_{\text {flex }}\right)^{T}$ was then pre-processed in such a way as to predict the human-commanded equilibrium position $q_{0}^{*}$ and stiffness $k^{*}$ of the wrist. Specifically, as a measure of stiffness we used the co-contraction level

$$
k^{*}=g_{k} \min \left(a_{e x t}, a_{f l e x}\right)+c_{k},
$$

and as a measure of equilibrium positions we used the signal difference

$$
q_{0}^{*}=g_{q_{0}}\left(a_{e x t}-a_{\text {flex }}\right)
$$

where $g_{q_{0}}$ and $g_{k}$ are gain parameters that scale the effect of the EMG on the commanded stiffness and equilibrium positions, and $c_{k}=k_{m i n}$ is an offset parameter that ensures the commanded stiffness never falls below the minimum achievable stiffness for the VSA.

In Fig. 5 we show results over $20 s$ of operation. These are broken into three phases: (i) alternating left-right hand movement with low stiffness (muscles relaxed), (ii) alternation between low and high stiffness at $q=0$ (relaxed/co-contracted muscles, respectively) and, (iii) alternating left-right hand movement with high stiffness (muscles co-contracted). The first and last conditions are indicated by the shaded regions in the plots.

As can be seen, during phase (i), the EMG signals indicate alternating activation between the two muscles, resulting in a left-right movement of the desired equilibrium position. The robot tracks this movement with considerable accuracy, albeit with a slight time delay, which we attribute to the limited speed of the servos used in the device. We also note that there is some small level of co-activation in the EMG signals even in this relaxed state: this is also tracked as small increases in stiffness. During phase (ii), the hand remains at the rest position $q=0$ and the operator co-contracts twice. As can be seen, this causes two spikes in the stiffness profile, which are also accurately tracked. It is interesting to note in the plot of the commands to the MACCEPA, the controller primarily relies on the second (pre-tensioning) motor for this, since there is a linear dependence between $u_{2}$ and stiffness at equilibrium. Finally, during phase (iii) we again see good tracking of the equilibrium position with increased overall stiffness, despite the relatively high noise in the recorded EMG. The performance of the controller can be further verified in the accompanying video.

\section{Constrained-stiffness Optimal Control}

In our final investigation, we briefly illustrate the use of our approach for analysing the benefits of VSAs over traditional, fixed-stiffness actuators. For this, we use the method described in Sec. III-D to compare the optimal behaviour of the three example 1-DOF VSAs described in Sec. II in a ball-hitting task, similar to that described in [6]. The set-up was as follows.

We used the iterative Local Quadratic Regulator (iLQR) algorithm [14] to seek local optimal feedback controllers (OFCs) that minimise the objective

$$
J=w_{1}\left(q(T)-q^{*}\right)^{2}-w_{2} \dot{q}(T)+\int_{0}^{T} w_{3} \tau^{2} d t
$$

where $q^{*}=30^{\circ}$ is the target angle (corresponding to the angle of impact with the ball), $\tau$ is applied joint torque and $w_{i}, i \in\{1,2,3\}$ are weight factors that determine the relative importance of the three terms. These, respectively, correspond to (i) minimising the distance to the target (ball) at the time of impact $T$, (ii) maximising the angular velocity at $T$, and (iii) minimising effort during the movement.

We performed the optimisation for each of the VSAs, (i) with no constraint, and (ii) with the constraint that the stiffness must remain fixed throughout the movement at the initial value $k_{0}$. In the latter case, the constraint is enforced in the manner described in Sec. III-D by specifying $k^{*}(t)=k_{0}$ throughout the movement.

In Fig. 6 we plot the stiffness profile, joint positions and joint velocities generated by the OFCs for the three VSAs, under the two conditions.

The first thing we see, looking at Fig. 6 (top row) is that the stiffness constraint is successfully enforced with good accuracy, albeit with some small deviation from the desired stiffness toward the end of the movement. We attribute these small errors to constraint drift, and the online feedback not fully compensating for the non-linear dynamics (both of which factors can be exacerbated in high velocity movements, as here). These errors, however, are negligible in comparison to the overall variation in stiffness seen in the unconstrained stiffness profiles (compare light red and black lines in Fig. 6, top row).

Looking at the behaviour (Fig. 6, middle and bottom rows), we see that if the stiffness is allowed to vary freely (light red lines), the OFCs exploit this additional degree of redundancy to improve task performance compared to the case that the stiffness is fixed (black lines). For example, comparing the variable against the fixed stiffness behaviour we see that (i) the variable stiffness controllers come closer 


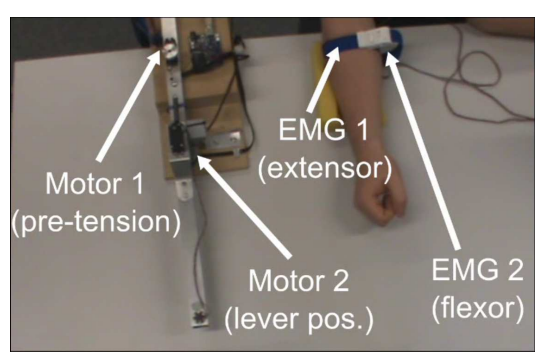

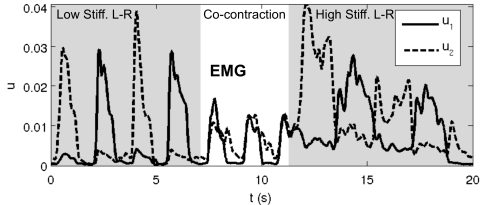

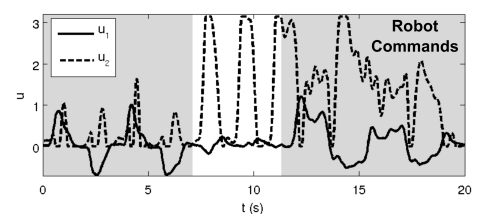

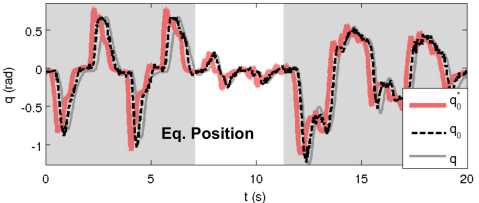

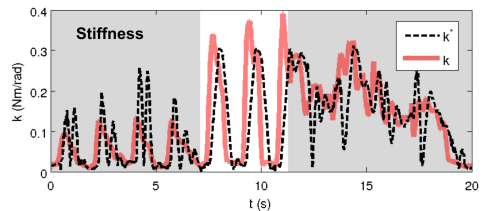

Fig. 5. Teleoperated control of equilibrium position and stiffness on the MACCEPA. Shown are human EMG signals, equilibrium position, stiffness, robot motor commands. Light-red and black-dashed lines denote desired trajectories (predicted from the human data via (21) \& (22)) and realised trajectories, respectively.
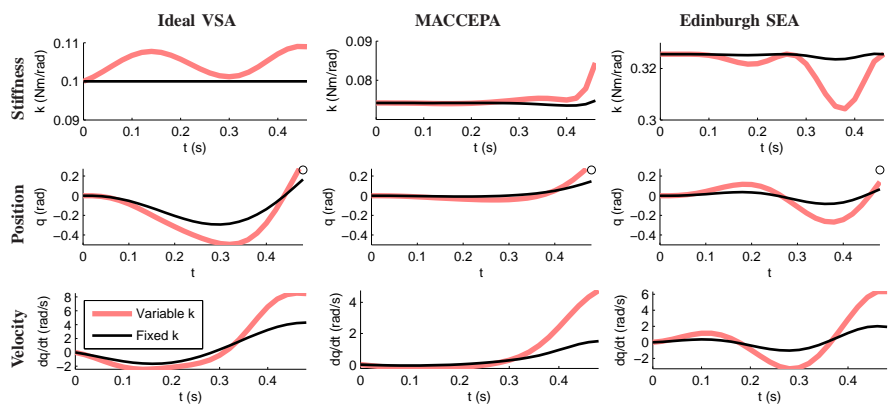

Fig. 6. Optimised ball hitting behaviour for the three single-joint VSAs when stiffness is (i) fixed (constrained) to the initial value (black), and (ii) allowed to vary freely (light red). Shown are: joint stiffness $k$ (top row), joint positions $q$ (middle row) and joint velocity $\dot{q}$ (bottom row) during the movement. The position of the target (ball) is indicated by ' $\mathrm{o}$ '.

\begin{tabular}{|c|c|c|}
\hline & Fixed $k$ & Variable $k$ \\
\hline Ideal 1-link VSA & -0.242372 & -0.489173 \\
\hline MACCEPA & -0.075511 & -0.274747 \\
\hline Edinburgh SEA & -0.076414 & -0.356527 \\
\hline
\end{tabular}

TABLE II

COST INCURRED BY OPTIMAL FEEDBACK CONTROLLERS FOR THE THREE SINGLE-JOINT VSAS PERFORMING THE BALL-HITTING TASK UNDER CONDITIONS OF FIXED OR VARIABLE STIFFNESS.

to the target (Fig. 6, middle row) and (ii) their end-time velocity is greater (Fig. 6, bottom row). The benefit of variable stiffness is further confirmed by comparing the cost incurred during the movement under the different conditions, which is uniformly lower when the stiffness is allowed to vary (see Table II).

\section{CONCLUSION}

In conclusion, we have presented a novel model-based method for control of variable stiffness actuators using constraints on equilibrium positions and stiffness in task and joint space. The proposed approach is generic by its formulation, and can be applied to many different designs of variable stiffness devices for accurate tracking of desired stiffness and equilibrium position profiles. Furthermore, as shown in simulation and experiment, it is fast to compute and can be used with ease for online stiffness control, such as in the teleoperation setting explored here.

In future work, we intend to exploit this method as a tool for (i) assessing in detail the benefits of variable stiffness actuation as compared to traditional, fixed stiffness actuators, and (ii) for evaluating methods for transfer of human impedance behaviour to artificial systems. Furthermore, we intend to explore extensions of the method, for example, to incorporate unilateral constraints, so that safety limits (such as limits on the maximum admissible stiffness) may be realised with arbitrary VSA hardware designs.

\section{ACKNOWLEDGEMENTS}

This work was funded by the EU Seventh Framework Programme (FP7) as part of the STIFF project.

\section{REFERENCES}

[1] Alin Albu-Schaeffer, Christian Ott, and Gerd Hirzinger. A unified passivity-based control framework for position, torque and impedance control of flexible joint robots. Int. J. Robotics Res, 26:23-39, 2007.

[2] David J. Braun and Michael Goldfarb. Eliminating constraint drift in the numerical simulation of constrained dynamical systems. Computer Methods in Appl. Mech. and Engineering, 198:3151-3160, 2009.

[3] Pasquale Chiacchio, Stefano Chiaverini, Lorenzo Sciavicco, and Bruno Sicilian. Closed-loop inverse kinematics schemes for constrained redundant manipulators with task space augmentation and task priority strategy. Int. J. Robotics Res., 10:410-425, 1991.

[4] Gowrishankar Ganesh, Alin Albu-Schaeffer, Masahiko Haruno, Mitsuo Kawato, and Etienne Burdet. Biomimetic motor behavior for simultaneous adaptation of force, impedance and trajectory in interaction tasks. ICRA, 2010.

[5] R. Van Ham, B. Vanderborght, M. Van Damme, B. Verrelst, and D. Lefeber. MACCEPA, the mechanically adjustable compliance and controllable equilibrium position actuator: Design and implementation in a biped robot. Robotics and Auton. Systems, (10):761-768, 2007.

[6] M. Howard, D. Mitrovic, and S. Vijayakumar. Transferring impedance control strategies between heterogeneous systems via apprenticeship learning. Humanoids, 2010.

[7] Masazumi Katayama and Mitsuo Kawato. Virtual trajectory and stiffness ellipse during multijoint arm movement predicted by neural inverse models. Biol. Cybern., 69:353-362, 1993.

[8] Matteo Laffranchi, Nikos G. Tsagarakis, and Darwin G.Caldwell. A variable physical damping actuator (VPDA) for compliant robotic joints. ICRA, 2010.

[9] A. Liégeois. Automatic supervisory control of the configuration and behavior of multibody mechanisms. IEEE Trans. Sys., Man and Cybernetics, SMC-7:245-250, 1977.

[10] Alessandro De Luca, Riccardo Farina, and Pasquale Lucibello. On the control of robots with visco-elastic joints. ICRA, 2005.

[11] Djordje Mitrovic, Stefan Klanke, and Sethu Vijayakumar. Exploiting sensorimotor stochasticity for learning control of variable impedance actuators. Int. J. Robotics Res., 2010.

[12] Jan Peters, Michael Mistry, Firdaus Udwadia, Rick Cory, Jun Nakanishi, and Stefan Schaal. A unifying methodology for the control of robotic systems. IROS, 2005.

[13] Kenji Tahara, Suguru Arimoto, Masahiro Sekimoto, and Zhi-Wei Luo. On control of reaching movements for musculo-skeletal redundant arm model. Applied Bionics and Biomechanics, 6:11-26, 2009.

[14] Emanuel Todorov and Weiwei Li. A generalized iterative LQG method for locally-optimal feedback control of constrained nonlinear stochastic systems. American Control Conference, 2005.

[15] Sebastian Wolf and Gerd Hirzinger. A new variable stiffness design: Matching requirements of the next robot generation. ICRA, 2008. 